\title{
Botulinum toxin type A for Holmes tremor secondary to thalamic hemorrhage
}

\author{
Pamela Latino $^{1,2} \cdot$ Francesco E. Pontieri $^{1,2,3} \cdot$ Francesco Orzi $^{1,2} \cdot$ Morena Giovannelli $^{2}$
}

Received: 18 March 2015/ Accepted: 9 June 2015

(C) Springer-Verlag Italia 2015

\section{Dear Editor,}

Holmes tremor (HT) is a low-frequency rest and intentional tremor frequently affecting the upper limb. The tremor, typically aggravated by movements, may in addition show an intrinsic postural component. It develops weeks to months after the onset of focal lesions of the brainstem, cerebellum and thalamus [1]. The response to a number of pharmacological agents consistently employed in the clinical practice, including levodopa, dopamine agonists, levetiracetam, clonazepam, zonisamide and propranolol, is rather unsatisfactory [2]. In drug-refractory cases, thalamotomy or deep brain stimulation of the nucleus ventralis intermedius of the thalamus may prove useful, but neurosurgical approaches, although potentially beneficial, are limited or avoided for being invasive and expensive [2].

Herewith, we report on a subject suffering for HT secondary to thalamic hemorrhage. The patient showed a good response to treatment with botulinum toxin type A (BoNT-A).

A 45-year-old, right-handed man with a history of hypertension developed right hemiparesis (manual muscle testing-MMT—score $2 / 5$ in the right arm and leg),

Electronic supplementary material The online version of this article (doi:10.1007/s10072-015-2285-z) contains supplementary material, which is available to authorized users.

Morena Giovannelli

giovannelli.morena@gmail.com

1 Department of Neuroscience, Mental Health and Sensory Organs (NESMOS), Sapienza University, Rome, Italy

2 Unit of Neurology, Sant'Andrea Hospital, Via di Grottarossa, 1035-00189 Rome, Italy

3 IRCCS Santa Lucia Foundation, Rome, Italy superficial hemihypoesthesia and dysarthria due to a left thalamic hemorrhage that was diagnosed by brain MRI. Following the acute phase, the patient was transferred to a rehabilitation unit, where he showed a progressive improvement. One month later, muscle strength of the right limbs recovered to MMT score 4/5. Two months after stroke the almost complete recovery of the hemiparesis was counterbalanced by the onset of postural, kinetic and resting tremor of the right arm. The tremor was irregular, of large amplitude and slow frequency $(3-4 \mathrm{~Hz}$ ), mostly proximal (involving the deltoid, biceps and triceps brachii), and accompanied by slight athetoid movements of the right hand (video as supplementary material). The tremor interfered significantly with activities of daily living [TRG Essential Tremor Rating Assessment Scale (TETRAS) "Activities of daily living subscale" score $=32$; "Performance subscale" score $=13$ ]. A further MRI scan showed the expected evolution of the thalamic hemorrhage and no additional focal vascular lesions. The patient was diagnosed presenting HT and treated with levodopa (100 mg, t.i.d., for $1 \mathrm{month})$, clonazepam $(1 \mathrm{mg}$, t.i.d., for another month), and propranolol (40 mg, b.i.d., for a further month). The treatment had no benefit (see video, pretherapy session). He refused dopamine agonists and levetiracetam.

On the basis of previous studies reporting the effectiveness of BoNT-A for treatment of upper limb tremor [3], we planned to treat the patient with BoNT-A. Following approval by our local Ethics Committee, we initiated the treatment by performing local injections of BoNT-A (abobotulinumtoxinA) into the following muscles of the right arm: deltoid and triceps brachii (250 IU each) and biceps brachii (500 IU). Tremulous muscle activity was identified by muscle palpation and confirmed by electromyography. Treatment was performed without 
ultrasonography guide. BoNT-A injections were repeated at 3-month interval for a total of three sessions for a total follow-up period of 9 months. After the second session, the patient showed a significant reduction of tremor severity, in particular of resting and postural components, with marked improvement of daily activities such as feeding, drinking, dressing and writing (TETRAS "Activities of daily living subscale" score = 20; "Performance subscale" score $=8$ ) (video in supplementary material). The efficacy of both second and third injections of BoNT-A lasted more than 2 months. BoNT-A injections were not accompanied either by muscle weakness, as measured by MMT score, or by other significant side effect.

HT is a relatively rare condition caused by focal lesions in the brainstem, cerebellum or thalamus, causing dysfunction of the basal ganglia and cerebellofugal pathways $[1,4]$, thus concurring with the onset of resting, postural and kinetic tremor. In most cases, pharmacological therapy does not achieve satisfactory results [2]. The effectiveness of BoNT-A for treatment of upper limb tremor has been reported recently, with functional benefit in more than $60 \%$ of cases [3]. Moreover, BoNT-A has been recommended for treatment of refractory essential tremor of the upper limbs based on the results of class II studies [3]. Ahn et al. [5] reported recently the efficacy of BoNT-A injection in a case with HT secondary to pontine hemorrhage. Consistent with the previous report [5], our patient experienced a satisfactory improvement of resting and postural tremor together with attenuation of intentional tremor. Moreover, the present report suggests that infiltration of proximal muscles improves motor performances even at distal level. Such an extended efficacy should be taken into account when there is concern for the occurrence of muscle weakness frequently complicating BoNT-A infiltration of the forearm muscles and/or intrinsic muscles of the hand.

In conclusion, this case suggests that BoNT-A is effective for treatment of HT. If the efficacy and safety of BoNT-A will be confirmed by further studies on larger series, BoNT-A might well be considered as therapeutic option for the HT unresponsive to oral drugs.

Conflict of interest The authors report no sources of fundings and no conflict of interests.

Informed consent Informed consent was obtained from the participant of the study.

\section{References}

1. Deuschl G, Bergman H (2002) Pathophysiology of nonparkinsonian tremor. Mov Disord 17(suppl. 3):S41-S48

2. Puschmann A, Wszolek ZK (2011) Diagnosis and treatment of common forms of tremor. Semin Neurol 31:65-77

3. Kim SD, Yiannikas C, Mahant N, Vucic S, Fung VS (2014) Treatment of proximal upper limb tremor with botulinum toxin therapy. Mov Disord 29:835-838

4. Seidel S, Kasprian G, Leutmezer F, Prayer D, Auff E (2009) Disruption of nigrostriatal and cerebellothalamic pathways in dopamine responsive Holmes tremor. J Neurol Neurosurg Psychiatry 80:921-923

5. Ahn SY, Kim DA, Park YO, Shin JH (2014) Effect of ultrasonography-guided botulinum toxin type a injection in Holmes tremor secondary to pontine hemorrhage: case report. Ann Rehabil Med 38:694-697 